$13^{\text {th }}$ International Conference on AEROSPACE SCIENCES \& AVIATION TECHNOLOGY, ASAT-13, May 26 - 28, 2009, E-Mail: asat@mtc.edu.eg Military Technical College, Kobry Elkobbah, Cairo, Egypt Tel : +(202) 24025292 - 24036138, Fax: +(202) 22621908

\title{
Performance Analysis of WCDMA System for LEO Satellite Channel
}

\author{
A. El-Mahdy ${ }^{*}$, A. Mahran ${ }^{* *}$ and S. Ibrahim ${ }^{* * *}$
}

\begin{abstract}
In a Low Earth Orbit (LEO) satellite mobile multimedia system, the multiple access scheme should be capable of supporting the necessary features that would facilitate the provision of multimedia services. Wideband Code Division Multiple Access (WCDMA) is an attractive multiple access scheme for mobile multimedia communications based on LEO satellites. In this paper, the performance of WCDMA system over LEO satellite channel is evaluated. The performance is measured in terms of the bit error probability. A mathematical derivation of the bit error probability is presented. A rake receiver with maximal ratio combining is considered in the analysis. The effect of the number of users and the processing gain on the performance of the WCDMA system is investigated.
\end{abstract}

Keywords: Wide Bband Code Division Multiple Access (WCDMA), Low Earth Orbit (LEO) Satellite, Frequency Selective Rayleigh Fading, Rake Receiver.

\section{Introduction}

In a Low Earth Orbit (LEO) satellite mobile multimedia system, the multiple access scheme should be capable of supporting the necessary features that would facilitate the provision of multimedia services [1, 2]. Wideband Code Division Multiple Access (WCDMA) is generally recognized as an attractive multiple access scheme for multimedia applications and features certain advantages like simultaneous support of multi-rate services, beneficial exploitation of multipath propagation, interference mitigation, and full frequency reuse. These features also make it an attractive multiple access scheme for mobile multimedia communications based on LEO satellites, especially if the satellite soft handoff and path diversity possibilities are exploited $[3,4]$.

There are many papers in the literature that discussed the performance evaluation of WCDMA systems over frequency-selective multipath fading channels but in this paper we will derive the error probability of such system in a LEO satellite channel. In [5] a user-friendly WCDMA simulator is built and used to investigate the performance of WCDMA system for different channel condition in a multi-user environment. A comparison between matched filtering and rake receiver performance and convolutional coding effects were studied. In [6] a "k-path" channel model was employed to examine the performance of WCDMA systems. The dependence of WCDMA system performance not only on the spread bandwidth but also on

\footnotetext{
Assoc. Prof, EAF, elmahdy77@hotmail.com

** Lecturer, EAF, a.mahran@gawab.com

**** Msc Student, EAF, sshawky78@yahoo.com
} 
radio propagation channel conditions was examined. The results show that the choice of the best spread bandwidth depends greatly on radio propagation channel conditions. The performances of different spreading factors have been analyzed in various channel conditions in [7]. Computer simulations were performed for the Rake receiver employing the Wiener filter as a channel estimator. A single user scenario has been used and the performance was also analyzed for two different Doppler frequencies corresponding to different speeds. The performance of WCDMA in multipath channels was evaluated in [8] based on the link level simulations with a Rake receiver in different multipath profiles. The effect of inter-path interference was analyzed and the possible gains from advanced receiver structures in canceling inter-path interference were evaluated. Also, the multipath diversity gain was studied for high bit rates. A hybrid FDMA/CDMA scheme operating over a frequency selective Rayleigh fading channel was described and analyzed in [9]. The performance of the hybrid- system is then compared with that of a wideband CDMA system occupying the same total bandwidth. Both coherent and non-coherent modulation formats were investigated; it was found that, for coherent modulation with a RAKE receiver, wideband CDMA has greater capacity than does the hybrid.

The following papers covered a performance analysis of the WCDMA systems over fading channels. Reference [10] presented a mathematical model for the statistics of WCDMA signals which experience multipath fading. The statistics were obtained from numerically generated signals, and were then modeled with an offset non-central chi distribution with three degrees of freedom. In [11], the performance of the conventional Rake receivers using Maximum Ratio Combining (MRC) for a UMTS terrestrial Radio - Wideband Code Division Multiple Access (UTRA-WCDMA) downlink was investigated. Both perfect and non perfect multipath slowly Rayleigh fading channel estimations are considered. Exact bit error probabilities (BER) are derived, using quadratic forms and characteristic functions, for BPSK modulation.

References [12,13] discussed the performance of WCDMA systems over LEO satellite communication channels. The average bit error rate (BER) of a wideband DS-CDMA, land mobile LEO satellite communication system with an equal-gain RAKE receiver was derived in [12]. It considered an urban channel model, which is a combination of a narrowband model and a wideband tapped delay line model. The analytical results with BPSK modulation, validated by simulation, were provided to illustrate the achievable performance with both diversity and no diversity reception for various satellite elevation angles. Reference [13] presented a comparative system study and evaluates, by means of computer simulation, the BER performance of W-CDMA and OFDM operating in a LEO satellite environment. The most important characteristics of the LEO satellite communications channel were presented. The simulation results evaluating the uplink BER performance for a single user indicate that, although the BER performance of W-CDMA is slightly better than that of OFDM, the performance differences are rather small.

In this paper the LEO satellite channel model is considered and the performance analysis of a WCDMA system using an MRC-Rake receiver is derived. This paper is organized as follows. Section (II) covers the proposed system model of the WCDMA transmitter, LEO satellite channel model and the WCDMA receiver. In Section (III) the error probability is derived for BPSK modulation. Simulation results are discussed in Section (IV). 


\section{System Model}

\section{A. Transmitter}

For a binary WCDMA system with $K$ independent users simultaneously sharing a channel, each user is transmitting with power $P$ at a common carrier frequency $f_{c}=\omega_{c} / 2 \pi$, using a data rate $R_{b}=1 / T_{b}$ and a chip rate $R_{c}=1 / T_{c}$. The $k^{\text {th }}$ user $(k=1,2, \ldots, K)$ is assigned a unique code sequence $\left\{a_{k, j}\right\}$ of chip elements $(+1,-1)$, so that its code waveform is given by:

$$
a_{k}(t)=\sum_{j=-\infty}^{+\infty} a_{k, j} P_{T_{c}}\left(t-j T_{c}\right)=\sum_{j=1}^{N_{k}} a_{k, j} w_{k}^{c h}(j) C_{s c r}\left(j+m N_{k}\right) P_{T_{c}}\left(t-j T_{c}\right)
$$

where $N_{k}$ is the user specific spreading factor. The data symbols are spread by $N_{k}$-chip Walsh channelization codes $w_{k}(j)$ and further scrambled by a Gold code $C_{s c r}(j)$. The two codes are mixed in a single time-varying and user $k$ specific spreading code.

The function $P_{T}($.$) denotes the chip pulse of duration T$. We assume that $P_{T}($.$) is a unit$ rectangular pulse. The code sequence $\left\{a_{k, j}\right\}$ is assumed to be periodic with period equal to the processing gain $P G=T_{b} / T_{c}$. The data signal waveform $b_{k}(t)$ is given by

$$
b_{k}(t)=\sum_{j=-\infty}^{+\infty} b_{k, j} P_{T_{b}}\left(t-j T_{b}\right)=\sum_{j=1}^{M_{k}} b_{k, j} P_{T_{b}}\left(t-j T_{b}\right)
$$

It is binary phase-shift-keyed (BPSK) onto the carrier at $f_{c}$, which is then spread by that user's code sequence and transmitted over the channel. $M_{k}$ is the number of data symbols sent from user $k$. The resulting $k^{\text {th }}$ user's transmitted signal $s_{k}(t)$ is thus given by

$$
s_{k}(t)=\sqrt{2 P} a_{k}(t) b_{k}(t) \cos \left(\omega_{c} t\right)
$$

The composite transmitted signal $s(t)$ at the input of the channel can then be expressed as:

$$
s(t)=\sum_{k=1}^{K} \sqrt{2 P} a_{k}(t) b_{k}(t) \cos \left(\omega_{c} t\right)
$$




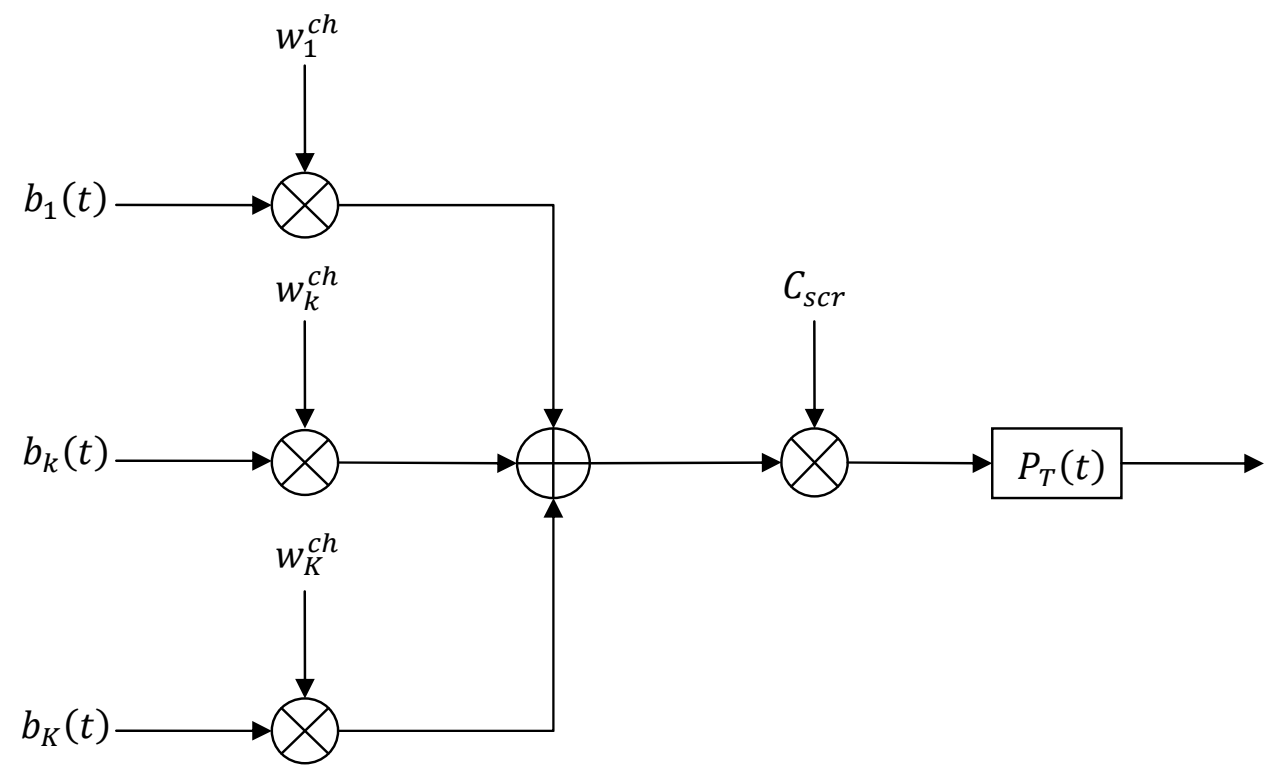

Figure (1) WCDMA transmitter architecture

\section{B. LEO Satellite Channel Model}

The LEO satellite communications channel exhibits both time variance due to the motion of the LEO satellite and/or mobile terminal, and frequency selectivity due to multipath propagation $[14,15]$. Thus, the LEO satellite channel is characterized by both Doppler spread and delay spread. It is assumed that the total Doppler spread is composed of two components, the first related to the motion of the satellite and the second to the motion of the mobile terminal [14]. There are a selection of sounding campaigns and ray-tracing results for the LEO satellite channel at frequencies around $2 \mathrm{GHz}$ [16] utilized by the satellite UMTS bands. The important conclusion from these works is that the LEO satellite channel is characterized by path losses much smaller than the equivalent losses of GEO satellites, and by delay spreads which typically range from 250 to $800 \mathrm{~ns}$. This range depends on whether there is line-ofsight (LOS) or non-LOS (NLOS) conditions between the LEO satellite and the mobile terminal as well as on the terrestrial propagation environment. In statistical terms, when a LOS path exists, the received signal amplitude is Ricean distributed, while in the case of NLOS the received signal amplitude follows a Rayleigh distribution $[14,15]$. The transmitted signal has a bandwidth which is much wider than the channel coherence bandwidth, and therefore undergoes frequency-selective fading. This type of fading is typically modeled by a linear filter, which for the $k^{\text {th }}$ user is characterized by a complex-valued low-pass equivalent impulse response [17-19]

$$
h_{k}(t)=\sum_{l=1}^{L_{p}} \alpha_{k, l} e^{-j \theta_{k, l}} \delta\left(t-\tau_{k,}\right)
$$

where $\delta($.$) is the Dirac delta function, l$ is the propagation path index, and $\left\{\alpha_{k, l}\right\}_{l=1}^{L_{p}},\left\{\theta_{k, l}\right\}_{l=1}^{L_{p}}$, and $\left\{\tau_{k, l}\right\}_{l=1}^{L_{p}}$ are the random path amplitudes, phases, and delays, respectively. We assume that the sets and $\left\{\alpha_{k, l}\right\}_{l=1}^{L_{p}},\left\{\theta_{k, l}\right\}_{l=1}^{L_{p}}$, and $\left\{\tau_{k, l}\right\}_{l=1}^{L_{p}}$ are mutually independent. In (5), $L_{p}$ is the number of resolvable paths (the first path is the reference path whose delay $\tau_{1}=0$ ) and is related to the ratio of the maximum delay spread $\tau_{\max }$ to the chip time duration $T_{c}$. 
We assume slow fading, so that $L_{p}$ is constant over time, and $\left\{\alpha_{k, l}\right\}_{l=1}^{L_{p}},\left\{\theta_{k, l}\right\}_{l=1}^{L_{p}}$, and $\left\{\tau_{k, l}\right\}_{l=1}^{L_{p}}$ are all constant over a symbol interval. If the different paths of a given impulse response are generated by different scatterers, they tend to exhibit negligible correlations [20, 21]. In this case it is reasonable to assume that the $\left\{\alpha_{k, l}\right\}_{l=1}^{L_{p}}$ are statistically independent random variables (RVs). We denote the fading amplitude of the $k^{\text {th }}$ user $l^{\text {th }}$ resolved path by $\alpha_{k, l}$, which is a RV whose mean-square value $\overline{\alpha_{k, l}^{2}}$ and it is assumed to be independent of $k$ and is denoted by $\Omega_{l}$.

After passing through the fading channel, the signal is perturbed by additive white Gaussian noise (AWGN) with a one-sided power spectral density denoted by $N_{0}$. The AWGN is assumed to be independent of the fading amplitudes $\left\{\alpha_{k, l}\right\}_{l=1}^{L_{p}}$. Hence, the instantaneous SNR per bit of the $l^{\text {th }}$ channel is given by $\gamma_{k, l}=\left(\alpha_{k, l}^{2} E_{b}\right) / N_{0}$ where $E_{b}$ is the energy per bit, and the average SNR per bit of the $l^{\text {th }}$ channel is given by $\overline{\gamma_{l}}=\left(\Omega_{l} E_{b}\right) / N_{0}$.

\section{Receiver}

With multipath propagation, it follows from (5) and (4) that the received signal $r(t)$, whose signal component is the time convolution of $s(t)$ and $h(t)$, may be written as

$$
r(t)=\sqrt{2 P} \sum_{k=1}^{K} \sum_{l=1}^{L_{p}} \alpha_{k, l} a_{k}\left(t-\tau_{k, l}\right) b_{k}\left(t-\tau_{k, l}\right) \cos \left(\omega_{c}\left(t-\tau_{k, l}\right)+\theta_{k, l}\right)+n(t)
$$

where $n(t)$ is the receiver AWGN random process. We consider an L-branch (finger) MRC receiver. For equally likely transmitted symbols, the total conditional SNR per symbol $\gamma_{t}$ at the output of the MRC combiner is given by [22]

$$
\gamma_{t}=\sum_{l=1}^{L_{p}} \gamma_{l}
$$

For coherent binary signals the conditional BER, $P_{b}\left(E \mid\left\{\gamma_{l}\right\}_{l=1}^{L_{p}}\right)$, is given by

$$
P_{b}\left(E \mid\left\{\gamma_{l}\right\}_{l=1}^{L_{p}}\right)=Q\left(\sqrt{2 g \gamma_{t}}\right)
$$

where $g=1$ for coherent BPSK [23], and $Q($.$) is the Gaussian Q-function. Our goal is to$ evaluate the performance of the system in terms of users' average BER, and for this purpose the conditional BER (8) has to be statistically averaged over the random parameters $\left\{\gamma_{l}\right\}_{l=1}^{L_{p}}$. To solve this problem, the MGF-based approach will be used.

\section{Average Bit Error Rate of Binary Signals}

The Gaussian-Q function can be expressed as

$$
Q(x)=\frac{1}{\pi} \int_{0}^{\pi / 2} \exp \left(-\frac{x^{2}}{2 \sin ^{2} \theta}\right) d \theta
$$


Using this alternative representation of the Gaussian-Q function in (8), the conditional BER may be rewritten in a more desirable product form given by

$$
P_{b}\left(E \mid\left\{\gamma_{l}\right\}_{l=1}^{L_{p}}\right)=\frac{1}{\pi} \int_{0}^{\frac{\pi}{2}} \exp \left(-\frac{g \gamma_{t}}{\sin ^{2} \phi}\right) d \phi=\frac{1}{\pi} \int_{0}^{\frac{\pi}{2}} \prod_{l=1}^{L_{p}} \exp \left(-\frac{g \gamma_{l}}{\sin ^{2} \phi}\right) d \phi
$$

This form of the conditional BER is more desirable since we can first independently average over the individual statistical distributions of the $\gamma_{l}$ terms, and then perform the integral over $\phi$, as described in more detail below.

To obtain the unconditional BER, $P_{b}(E)$, when multichannel reception is used, we must average the multichannel conditional BER, $P_{b}\left(E \mid\left\{\gamma_{l}\right\}_{l=1}^{L_{p}}\right)$, over the joint PDF of the instantaneous SNR sequence $\left\{\gamma_{l}\right\}_{l=1}^{L_{p}}$, namely, $p_{\gamma_{1}, \gamma_{2} \ldots \gamma_{L_{p}}}\left(\gamma_{1}, \gamma_{2} \ldots \gamma_{L_{p}}\right)$. Since the RVs $\left\{\gamma_{l}\right\}_{l=1}^{L_{p}}$ are assumed to be statistically independent, then $p_{\gamma_{1}, \gamma_{2} \ldots \gamma_{L_{p}}}\left(\gamma_{1} . \gamma_{2} \ldots \gamma_{L_{p}}\right)=$ $\prod_{l=1}^{L_{p}} p_{\gamma_{l}}\left(\gamma_{l}\right)$, and the averaging procedure results in

$$
P_{b}(E)=\int_{0}^{\infty} \int_{0}^{\infty} \ldots \int_{0}^{\infty} P_{b}\left(\left\{\gamma_{l}\right\}_{l-0}^{L_{p}-1}\right) \prod_{l=1}^{L_{p}} p_{\gamma_{l}}\left(\gamma_{l}\right) d \gamma_{1} d \gamma_{2} \ldots d \gamma_{L_{p}}
$$

Using the alternative product form representation of the conditional BER (10) in (11) we have

$$
P_{b}(E)=\int_{0}^{\infty} \int_{0}^{\infty} \ldots \int_{0}^{\infty} \frac{1}{\pi} \int_{0}^{\frac{\pi}{2}} \prod_{l=1}^{L_{p}} \exp \left(-\frac{g \gamma_{l}}{\sin ^{2} \phi}\right) p_{\gamma_{l}}\left(\gamma_{l}\right) d \phi d \gamma_{1} d \gamma_{2} \ldots d \gamma_{L_{p}}{ }_{b}
$$

The integrand in (12) is absolutely integrable, and hence the order of integration can be interchanged. Thus, grouping terms of index $l$, we obtain

$$
P_{b}(E)=\frac{1}{\pi} \int_{0}^{\frac{\pi}{2}} \prod_{l=1}^{L_{p}} \mathrm{M}_{\gamma_{l}}\left(-\frac{g}{\sin ^{2} \phi}\right) d \phi
$$

where $\mathrm{M}_{\gamma_{l}}(s)$ is the Moment Generating Function (MGF) of SNR ber symbol $\gamma_{l}$.

If the fading is identically distributed with the same fading parameter and the same average SNR per bit $\bar{\gamma}$ for all $L_{p}$ channels, then (13) reduces to

$$
P_{b}(E)=\frac{1}{\pi} \int_{0}^{\frac{\pi}{2}}\left(\mathrm{M}_{\gamma}\left(-\frac{g}{\sin ^{2} \phi}\right)\right)^{L_{p}} d \phi
$$


For Rayleigh fading channel $\mathrm{M}_{\gamma_{l}}(s)=\left(1-s \overline{\gamma_{l}}\right)^{-1}$ taking into account that $g=1$ and substituting in (13) we get

$$
P_{b}(E)=\frac{1}{\pi} \int_{0}^{\frac{\pi}{2}} \prod_{l=1}^{L_{p}}\left(1+\frac{1}{\sin ^{2} \phi} \bar{\gamma}_{l}\right)^{-1} d \phi
$$

\section{Performance Analysis}

Let us consider the $k^{\text {th }}$ user's performance. The decision variable $r_{k}$ may be written as the sum of a desired signal component and three interference/noise components [24]

$$
r_{k}= \pm\left(\sum_{l=1}^{L_{p}} \alpha_{k, l}^{2}\right) \sqrt{E_{b}}+\sum_{l=1}^{L_{p}} \alpha_{k, l}\left(I_{s}+I_{M}+N\right)
$$

where $I_{S}$ is the self-interference component induced by the autocorrelation function of the $k^{\text {th }}$ user's spreading code, $I_{M}$ is the multiple-access interference (MAI) component induced by the other $K-1$ users on the desired user, and $N$ is a zero-mean AWGN component with variance $\sigma_{N}^{2}=N_{0} / 2 . I_{s}$ can be considered to be a zero-mean Gaussian RV with variance [24]

$$
\sigma_{S}^{2}=\frac{\Omega_{\mathrm{T}}-1}{2 P G} \Omega_{1} E_{b}
$$

where $\Omega_{\mathrm{T}}=\sum_{l=1}^{L_{p}} \Omega_{l} / \Omega_{1}$ can be interpreted as the normalized (to the first path) total average fading power. Similarly, under the standard Gaussian approximation (large numbers of users) $I_{M}$ can be modeled as a zero-mean Gaussian RV with variance [24]

$$
\sigma_{M}^{2}=\frac{2(\mathrm{~K}-1) \Omega_{\mathrm{T}}}{6 P G} \Omega_{1} E_{b}
$$

Under these assumptions $r_{k}$ may be considered to be a conditional Gaussian RV (conditioned on $\left\{\alpha_{k, l}\right\}_{l=1}^{L_{p}}$ with a conditional mean $E\left(r_{k} \mid\left\{\alpha_{k, l}\right\}_{l=1}^{L_{p}}\right)$ and a conditional variance $\operatorname{var}\left(r_{k} \mid\left\{\alpha_{k, l}\right\}_{l=1}^{L_{p}}\right)$ given by

$$
\begin{gathered}
E\left(r_{k} \mid\left\{\alpha_{k, l}\right\}_{l=1}^{L_{p}}\right)= \pm\left(\sum_{l=1}^{L_{p}} \alpha_{k, l}^{2}\right) \sqrt{E_{b}} \\
\operatorname{var}\left(r_{k} \mid\left\{\alpha_{k, l}\right\}_{l=1}^{L_{p}}\right)=\sum_{l=1}^{L_{p}} \alpha_{k, l}^{2}\left(\sigma_{N}^{2}+\sigma_{S}^{2}+\sigma_{M}^{2}\right)
\end{gathered}
$$

Assuming that the data bits +1 or -1 are equiprobable, the $k^{\text {th }}$ user conditional SNR, $\operatorname{SNR}\left(\left\{\alpha_{k, l}\right\}_{l=1}^{L_{p}}\right)$, is given as 


$$
\operatorname{SNR}\left(\left\{\alpha_{k, l}\right\}_{l=1}^{L_{p}}\right)=\frac{E\left(r_{k} \mid\left\{\alpha_{k, l}\right\}_{l=1}^{L_{p}}\right)}{2 \operatorname{var}\left(r_{k} \mid\left\{\alpha_{k, l}\right\}_{l=1}^{L_{p}}\right)}=\left(\sum_{l=1}^{L_{p}} \alpha_{k, l}^{2}\right) \frac{E_{b}}{N_{e}}
$$

where $N_{e} / 2$ is the equivalent two-sided interference plus noise power spectral density defined as

$$
\frac{N_{e}}{2} \triangleq \sigma_{N}^{2}+\sigma_{S}^{2}+\sigma_{M}^{2}=\left(\frac{2(\mathrm{~K}+1) \Omega_{\mathrm{T}}-3}{6 P G}\right) \Omega_{1} E_{b}+\frac{N_{0}}{2}
$$

With $\overline{\gamma_{1}}=\left(\Omega_{1} \mathrm{E}_{\mathrm{b}}\right) / N_{0}$ the average received SNR per bit corresponding to the first path. Form (21) and (22) and since we are assuming BPSK modulation, the average BER performance expression obtained in (15) applies here by replacing $N_{0}$ with $N_{e}$, or equivalently, replacing the average SNR per bit of the $l^{\text {th }}$ path $\overline{\gamma_{l}}=\left(\Omega_{l} \mathrm{E}_{\mathrm{b}}\right) / N_{0}$ by

$$
\bar{\gamma}_{l, e}=\frac{\Omega_{1} \mathrm{E}_{\mathrm{b}}}{N_{e}}=\bar{\gamma}_{l}\left[1+\left(\frac{2(\mathrm{~K}+1) \Omega_{\mathrm{T}}-3}{3 P G}\right) \overline{\gamma_{1}}\right]^{-1}
$$

Thus by substituting from (22) in (15) we get

$$
P_{b}(E)=\frac{1}{\pi} \int_{0}^{\frac{\pi}{2}} \prod_{l=1}^{L_{p}}\left(1+\frac{1}{\sin ^{2} \phi} \overline{\gamma_{l}}\left[1+\left(\frac{2(\mathrm{~K}+1) \Omega_{\mathrm{T}}-3}{3 P G}\right) \overline{\gamma_{1}}\right]^{-1}\right)^{-1} d \phi
$$

This integral will be evaluated using numerical methods by taking samples of $\phi$ and choosing the values of $\mathrm{K}, \mathrm{PG}, \overline{\gamma_{l}}$ and $\Omega_{\mathrm{T}}$. The following section shows the numerical results of this probability of error.

\section{Numerical Results}

The performance of the WCDMA system was evaluated numerically over the LEO satellite channel model for different parameters. The parameters used to evaluate the numerical results are as follows. The channel bandwidth is $\mathrm{B}=5 \mathrm{MHz}$, the maximum delay spread of the LEO satellite channel ranges from 250 to $800 \mathrm{~ns}$. The channel has 5 taps of gains of [0 -3 -6 - - - -12] and delays of [0 260e-9 521e-9 781e-9 989e-9]. The frame duration is $10 \mathrm{~ms}$ and the user data rate is $120 \mathrm{kbps}$. The user velocity is $20 \mathrm{~km} / \mathrm{h}$. The signal to noise ratio is defined as $E_{b} /$ No. In Figure (2), the performance of the WCDMA system for different number of users $\mathrm{K}$ is plotted for a fixed value of processing gain $(\mathrm{PG}=63)$. The figure shows that as number of users increases, the performance of the WCDMA system degrades. This is due to the increase of MAI. For example, at $\mathrm{BER}=10^{\wedge}-3$, the system with $\mathrm{K}=5$ has a performance loss about $5 \mathrm{~dB}$ from the system with $\mathrm{K}=1$. The effect of processing gain $(\mathrm{PG})$ on the performance of the system is illustrated in Figure (3). The results show that the system performance is improved as the PG increases. This is because as the PG increases, the cross correlation properties of the code are enhanced which causes enhancement in the system performance. 
Paper: ASAT-13-CM-22

\section{Conclusions}

In this paper, the performance of WCDMA system over the LEO satellite channel is investigated. The bit error probability of the WCDMA receiver with MRC is derived. It has been shown that the system performance degrades rapidly as the number of users increases. This is due to the increase of MAI. Moreover, when the processing gain (PG) is increased, the system performance is enhanced.

\section{References}

[1] A. Jamalipour, "Mobile Satellite Communications", Artech House, 1998.

[2] Ganz, A.; Yebin Gong; Bo Li, "Performance study of low Earth-orbit satellite systems," Communications, IEEE Transactions on , vol.42, no.234, pp.1866-1871, Feb/Mar/ Apr 1994.

[3] A. J. Viterbi, "CDMA Principles of Spread Spectrum Communication", AddisonWesley, 1995.

[4] Adachi, F.; Sawahashi, M.; Suda, H., "Wideband DS-CDMA for next-generation mobile communications systems ," Communications Magazine, IEEE , vol.36, no.9, pp.56-69, Sep 1998.

[5] Karamchand Babu, A.R.; Abdul Rashid, Z.A., "Performance evaluation of wideband CDMA air interface," Research and Development, 2002. SCOReD 2002. Student Conference on , vol., no., pp. 145-148, 2002.

[6] Hara, Y.; Morikawa, H.; Mizumachi, M., "Multipath fading effects on the performance of wideband CDMA systems," Universal Personal Communications. 1995. Record., 1995 Fourth IEEE International Conference on , vol., no., pp.620-624, 6-10 Nov 1995.

[7] Reyaz, T.; Burr, A., "Performance analysis of different spreading factors in various channel conditions with channel estimation for uplink WCDMA," Personal, Indoor and Mobile Radio Communications, 2005. PIMRC 2005. IEEE 16th International Symposium on , vol.2, no., pp.1204-1208 Vol. 2, 11-14 Sept. 2005.

[8] Holma, H.; Heiska, K., "Performance of high bit rates with WCDMA over multipath channels ," Vehicular Technology Conference, 1999 IEEE 49th , vol.1, no., pp.25-29 vol.1, Jul 1999.

[9] Eng, T.; Milstein, L.B., "Comparison of hybrid FDMA/CDMA systems in frequency selective Rayleigh fading," Selected Areas in Communications, IEEE Journal on , vol.12, no.5, pp.938-951, Jun 1994.

[10] Cheol-Sung Kim; Dong-Jin Oh; Milstein, L.B., "Statistical modeling of W-CDMA signals for use over frequency-selective multipath channels," Communications, IEEE Transactions on, vol.52, no.1, pp. 28-30, Jan. 2004.

[11] Guenach, M.; Vandendorpe, L., "Performance analysis of conventional RAKE receivers with channel estimation for a BPSK WCDMA downlink," Communications and Vehicular Technology, 2000. SCVT-200. Symposium on , vol., no., pp.194-201, 2000.

[12] Zhang Wei; Kot, A.C.; Li, K.H., "Performance analysis of a W-CDMA based land mobile satellite communication system with diversity combining over fading channels," Vehicular Technology Conference, 1999 IEEE 49th , vol.1, no., pp.156-160 vol.1, Jul 1999.

[13] Papathanassiou, A.; Salkintzis, A.K.; Mathiopoulos, P.T., "A comparison study of the uplink performance of W-CDMA and OFDM for mobile multimedia communications via LEO satellites," Personal Communications, IEEE, vol.8, no.3, pp.35-43, Jun 2001.

[14] Davis, L.M.; Collings, I.B.; Evans, R.J., "Estimation of LEO satellite channels," Information, Communications and Signal Processing, 1997. ICICS., Proceedings of 1997 International Conference on, vol.1, no., pp.15-19 vol.1, 9-12 Sep 1997. 
[15] Lutz, E.; Cygan, D.; Dippold, M.; Dolainsky, F.; Papke, W., "The land mobile satellite communication channel-recording, statistics, and channel model," Vehicular Technology, IEEE Transactions on, vol.40, no.2, pp.375-386, May 1991.

[16] Dottling, M.; Didascalou, D.; Wiesbeck, W., "Wideband channel modeling and diversity techniques for satellite-UMTS," Vehicular Technology Conference, 1999. VTC 1999 - Fall. IEEE VTS 50th, vol.5, no., pp.2770-2774 vol.5, 1999.

[17] Biyari, K.H.; Lindsey, W.C., "Binary communications through noisy, non-Gaussian channels," Information Theory, IEEE Transactions on , vol.40, no.2, pp.350-362, Mar 1994.

[18] Turin, G.L.; Clapp, F.D.; Johnston, T.L.; Fine, S.B.; Lavry, D., "A statistical model of urban multipath propagation," Vehicular Technology, IEEE Transactions on , vol.21, no.1, pp. 1-9, Feb 1972.

[19] H. Suzuki, "A statistical model for urban multipath propagation," IEEE Trans. Commun., vol. COM-25pp. 673-680, July 1977.

[20] Hashemi, H., "Impulse response modeling of indoor radio propagation channels," Selected Areas in Communications, IEEE Journal on , vol.11, no.7, pp.967-978, Sep 1993.

[21] Abbas, S.A.; Sheikh, A.U., "A geometric theory of Nakagami fading multipath mobile radio channel with physical interpretations," Vehicular Technology Conference, 1996. 'Mobile Technology for the Human Race'., IEEE 46th , vol.2, no., pp.637-641 vol.2, 28 Apr-1 May 1996.

[22] G. L. Stuber, "Principles of Mobile Communications," Norwell, MA: Kluwer Academic Publishers, 1996.

[23] M. K. Simon, S. M. Hinedi, and W. C. Lindsey, "Digital Communication TechniquesSignal Design and Detection,”. Englewood Cliffs, NJ: PTR Prentice-Hall, 1995.

[24] Eng, T.; Milstein, L.B., "Coherent DS-CDMA performance in Nakagami multipath fading," Communications, IEEE Transactions on , vol.43, no.234, pp.1134-1143, Feb/Mar/Apr 1995.

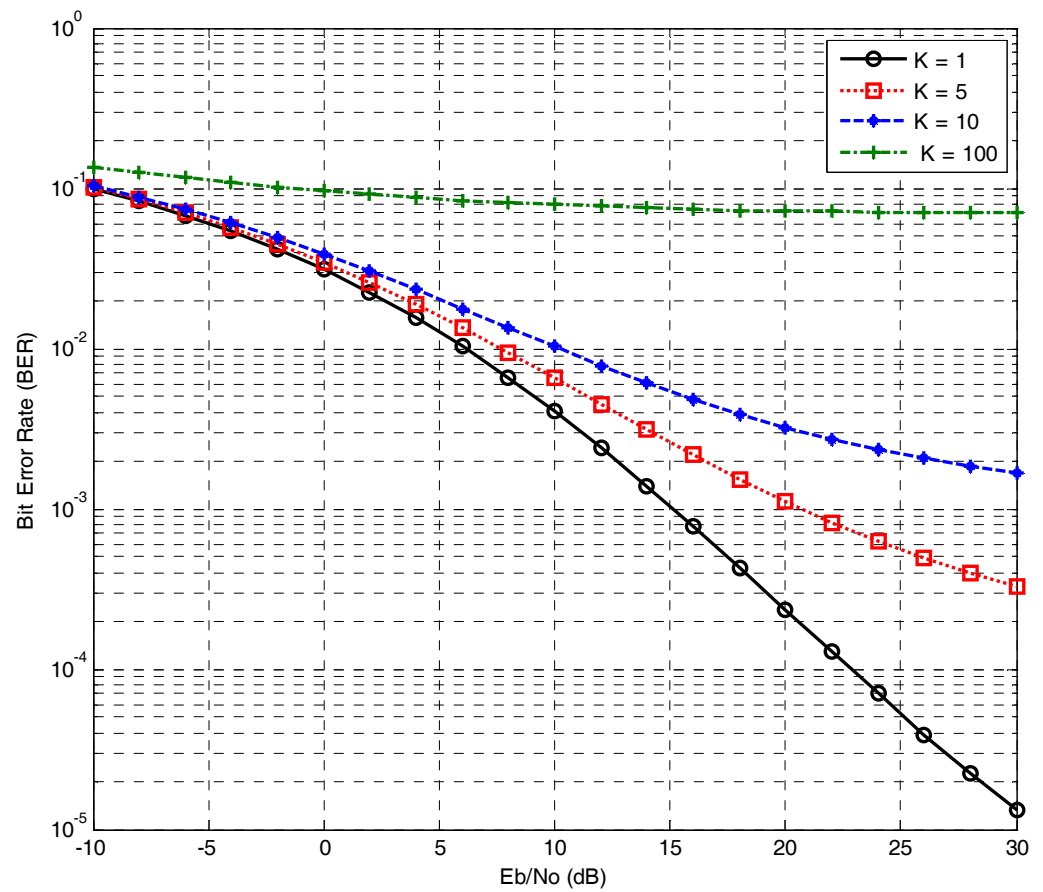

Figure (2) WCDMA system performance over LEO Satellite channel for different values of users (K) and fixed Processing Gain (PG = 63) 


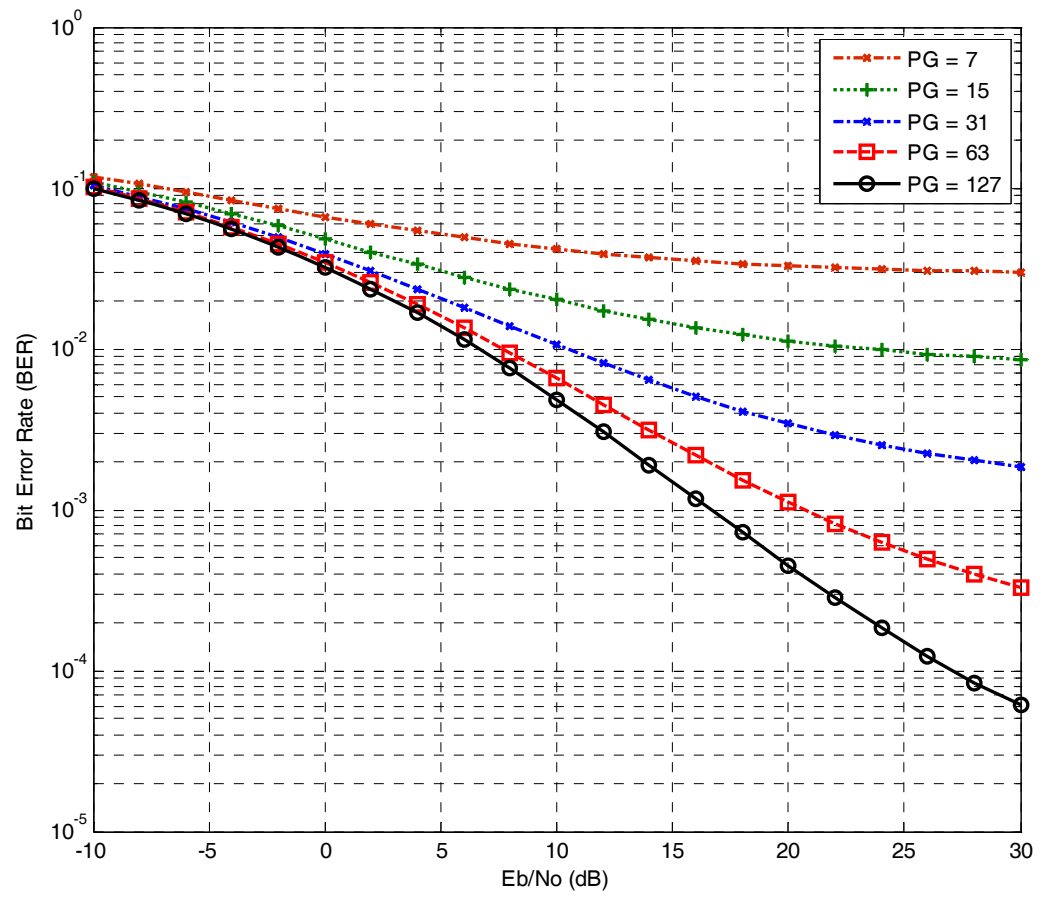

Figure (3) WCDMA system performance over LEO Satellite channel for different values of Processing Gain (PG) and fixed Number of Users $(K=5)$ 